\title{
Prevention of coronary heart disease
}

\section{Summary of the Report of a Joint Working Party of the Royal College of Physicians of London and the British Cardiac Society on Prevention of Coronary Heart Disease*}

British Medical fournal, 1976, 1, 881-882

The aim of the working party has been to formulate the best possible advice which can at present be given to medical practitioners towards the prevention of coronary heart disease (CHD). There is considerable evidence that the causes of $\mathrm{CHD}$ are largely environmental and are rooted in the modern affluent way of life. CHD risk factors such as cigarette smoking, physical inactivity, obesity, and high plasma lipid concentrations reflect aspects of our social behaviour. The working party's report ${ }^{1}$ is particularly concerned with those risk factors that can be modified.

The risk of CHD varies according to the total burden of risk factors present, and the recommendations emphasise this multifactorial concept of risk. When dealing with an individual the overall degree of risk of CHD must be considered rather than deciding whether any particular factor has reached a "critical" level requiring treatment. The recommended measures carry the reasonable hope of conferring some benefit to the community, and none of them costs as much as inaction.

\section{Risk factors and recommendations}

Diet-Dietary recommendations for the whole community involve a reduction in the amount of saturated fats and partial substitution by

* Members of the working party were: Sir Cyril Clarke, Professor J F Goodwin, Professor A G Shaper (chairman), Dr K P Ball, Professor J K Lloyd, Dr M F Oliver, Professor G A Rose, Dr W Somerville, Professor A S Truswell, and Dr R W D Turner; Dr M Ashley-Miller, observer from the Scottish Home and Health Department; Dr Gillian Ford, observer from the Department of Health and Social Security; Professor J S McCormick, representing the Royal College of General Practitioners; and Dr V M Hawthorne, representing the Faculty of Community Medicine. polyunsaturated fats. Where plasma lipid concentrations indicate a particularly high risk, or where other risk factors are concurrently present, the dietary recommendations should be followed more strictly. Widespread screening for plasma lipid levels is not recommended but estimations should be carried out in certain groups known to be at high risk of CHD. Maintaining a desirable weight is important, as obesity is commonly associated with other more potent risk factors for CHD. Weight reduction should be based on a decrease in all the dietary components; sugar and alcohol are recognised as common sources of excess energy intake. A combination of exercise and diet is strongly recommended.

Smoking-Every effort should be made to discourage cigarette smoking, particularly in the young. Doctors and other health workers should set an example, and less harmful methods of smoking should be advised for those who are unwilling to stop.

Blood pressure-Blood pressure should be recorded for every patient, using the opportunities provided by any consultation. In those with even moderately raised blood pressures the control of other risk factors (cigarette smoking, diet, physical inactivity) is important. Treatment of raised blood pressure is at present justified on the grounds of reducing the risk of stroke and other complications, but its effect on CHD risk is not yet established.

Physical activity-Physical activity should be encouraged at all ages and in both men and women. Few people need to consult their doctor before making a graded increase in their physical activity.

Stress-While acute stress may occasionally precipitate a heart attack, it is difficult to prove that chronic stress contributes to the development of CHD. The management of stress, whether it be domestic or occupational in origin, is a normal part of medical practice. Initiative, diligence, leadership, and hard work, especially in young people, should not be discouraged on the mistaken supposition that these qualities are indicators of future CHD.

Diabetes mellitus-Reversal of risk factors should form part of the care of diabetics, and dietary policy for individual diabetics should be determined as much by their plasma lipid concentrations as by the blood sugar response.

Oral contraceptives-Oral contraceptives constitute a negligible risk in women under the age of 40 years who have no risk factors for CHD, but they should be used with caution in women over 40 years, those with a family history of premature CHD, and those who are heavy cigarette smokers ( $>20 /$ day) or have other risk factors. 


\section{Children}

Measures recommended to prevent the development of CHD apply as much to children as to adults since all of the major risk factors found in adult life can occur during childhood. All those concerned with the care of children should be active in the prevention and management of these factors.

\section{General practice}

General practice should provide the main means of identifying those at high risk of CHD. Mass screening for CHD and its associated risk factors is not recommended, but the efforts of general practitioners should be supported by a general health education policy which involves hospital and community physicians and their supporting staff. Selective health examinations should be carried out in those groups known to be at high risk for CHD, and interested general practitioners should be encouraged to extend health examinations to other groups of patients when this can be done by using existing facilities and services.

\section{Wider implications of recommendations}

It was not the purpose of the working party to consider the implications of their recommendations for research or for government. Nevertheless, some areas may deserve further consideration by other bodies. With regard to the overall allocation of resources, the achievements of acute coronary care, coronary ambulances, and coronary artery surgery cannot bring about a major reduction in the overall burden of heart disease in the community. This must come from preventive measures.
Education-Bringing about the recommended changes in behaviour relating to diet, physical activity, and cigarette smoking will require the sustained education of the community at all levels, but particularly the young. A comprehensive public and professional educational programme will be needed together with the co-operation of food manufacturers, educational authorities, and the mass media. Apart from formal health education, much can be done by medical practitioners and other health workers who understand the problem and are prepared to provide positive advice to individuals and the community about the risk factors for CHD.

Smoking-The reduction of cigarette smoking will require fiscal measures by the government and the control of advertising. These measures may be at least as effective as general health education and possibly more so.

Diet-There are considerable implications in the dietary recommendations for national food policy, for the producers and the manufacturers of food, and for the regulations concerning food labelling. Nutrition practices and catering in schools, hospitals, the armed Forces, and other organisations may have to be reviewed.

Physical activity-Any improvement in patterns of physical activity can come only from leisure-time pursuits. Adequate and convenient recreational facilities are required if the recommendations are to be effective.

\section{Reference}

1 Joint Working Party, Fournal of the Royal College of Physicians of London, 1976, 10, 213.

\title{
Today's Treatment
}

\section{Psychological Medicine}

\section{Drugs used in psychological medicine: pharmacological basis of treatment}

\author{
JOHN C GILBERT
}

\section{Depression}

\section{MONOAMINE OXIDASE INHIBITORS}

A wide spectrum of drugs is used to treat psychological disorders and a strong element of serendipity has often triggered major discoveries in this field. For example, searches for new analgesic drugs led to the discovery of a potent antipsychotic agent, haloperidol, and it seems almost bizarre that studies of an antihelminthic drug should lead to introducing that drug, disulfiram, for treating alcoholism. For some of the drugs, after years of clinical use fully justified by empirical observations, laboratory studies have thrown light on mechanisms of action and in several cases relatively specific pharmacological receptors have been implicated. For most drugs, however, it is difficult to postulate one mechanism of action with confidence.

\footnotetext{
Department of Pharmacology, University of Aberdeen, Aberdeen AB9 2ZD

JOHN C GILBERT, PHD, reader in pharmacology
}

The first controlled clinical trial of a drug for treating depression appears to have been started some five years after the chance observation that patients suffering from tuberculosis who were treated with iproniazid experienced elevation of mood. During the intervening five years the powerful tranquillising effect of reserpine was found to be accompanied by central catecholamine and serotonin depletion, and, significantly, reserpine sedation was reversed by monoamine oxidase (MAO) inhibitors, drugs which caused a rise in the monoamine content of the brain. The discovery of MAO inhibitor antidepressants highlights the importance of monoamine metabolism in the brain and the manner in which pharmacological and neurochemical studies may lead to a rational approach to treatment.

Noradrenaline is synthesised in neurons of the brain from the amino-acid tyrosine via another important monoamine dihydroxyphenylalanine (DOPA), and after synthesis it becomes stored in synaptic vesicles within the nerve terminals. When a wave of depolarisa- 